\title{
Prevalence and risk factors of hyperkalemia early period after kidney transplantation
}

\author{
Sua Lee ${ }^{1}$, Kyeong Min Kim${ }^{1}$, Mi-Hyeong Kim², Jong Ho Shin ${ }^{1}$, Jihyang Lim³ ${ }^{3}$ Jeong-Kye Hwang ${ }^{2}$, Bum Soon Choi ${ }^{4}$, \\ Byung Soo Kim ${ }^{4}$, Tae Hyun Ban ${ }^{4}$ \\ 1Department of Internal Medicine-Nephrology, Eulji University Hospital, Daejeon, Korea
2Department of Surgery-Transplantation, The Catholic University of Korea, Eunpyeong St. Mary's Hospital, Seoul, Korea
${ }^{3}$ Department of Laboratory Medicine, The Catholic University of Korea, Eunpyeong St. Mary's Hospital, Seoul, Korea
${ }^{4}$ Department of Internal Medicine-Nephrology, The Catholic University of Korea, Eunpyeong St. Mary's Hospital, Seoul, Korea
}

Background: Electrolyte abnormality after kidney transplantation $(\mathrm{KT})$ is common complications owing to immunosuppressive agents, prophylactic antibiotics, anti-hypertensive agents and uncontrolled diabetes. Hyperkalemia is a fatal electrolyte abnormality that often leads to arrhythmia and sudden cardiac arrest. However, there are few studies on incidence and factors related to hyperkalemia after KT. We evaluated the prevalence of hyperkalemia and related factors for early period after transplantation. Methods: We analyzed database of patients who conducted KT in our institute from April 2019 to January 2021. Among 26 kidney transplant recipients (KTRs), 11 patients (42.3\%) had experienced hyperkalemia for 6 months after KT. We divided into two groups of normokalemic KTRs group $(n=15)$ and hyperkalemia KTRs group $(n=11)$ according to hyperkalemia. Hyperkalemia was defined as serum potassium over $5.1 \mathrm{mmol} / \mathrm{L}$ or use of potassium lowering agent owing to hyperkalemia. We compared clinical data between the two groups.

Results: The mean age of the patients was $52.0 \pm 9.5$ years with 15 patients $(57.7 \%)$ for male sex. Dialysis modality before transplantation was hemodialysis $(n=23,88.5 \%)$ in the most patients. A major cause of $K T$ was diabetes $(n=11,42.3 \%)$, then followed to hypertension $(n=8,30.8 \%)$ and chronic glomerulonephritis $(n=3,11.5 \%)$. The hyperkalemic KTRs group compared with the normokalemia KTRs group was older age ( 56.3 years vs. 48.9 years, $P=0.045)$, longer dialysis vintage (61.5 months vs. 29.6 months, $\mathrm{P}=0.045)$, more deceased donor $\mathrm{KT}(81.8 \%$ vs. $26.7 \%, \mathrm{P}=0.005)$ and more acute rejection $(54.5 \%$ vs. $93.3 \%, \mathrm{P}=0.05)$.

Conclusions: KTRs with older age and longer dialysis vintage need more stringent surveillance for hyperkalemia, especially if they received a transplant from deceased donor and treated acute rejection.

Corresponding author: Tae Hyun Ban

E-mail: deux0123@catholic.ac.kr

(c) The Korean Society for Transplantation

This is an Open Access article distributed under the terms of the Creative Commons Attribution Non-Commercial License (http://creativecommons.org/licenses/by-nc/4.0/) which permits unrestricted non-commercial use, distribution, and reproduction in any medium, provided the original work is properly cited. 